\title{
Der Fokus im Mittelfeld
}

\author{
Michael Richter (Kleve)
}

\begin{abstract}
In this article a proposal is made for the treatment of a construction which is known under the terminus "verschränkte Konstruktion" (Kvam 1982); it includes the so called "third construction". Example (1) shows a sentence with a syntactic complement of an infinite verb standing before the governing finite verb:
\end{abstract}

(1) ..., weil er dir versucht zu helfen.

The proposal is made that the NP on the "wrong" place (in (1) it is the indirect object dir) is a focus in the middlefield ("Mittelfeldfokus"). In the framework of the transformational grammar of Semantic Syntax (Seuren 1996) the sentence-predicate " $V_{\text {ATTR }}$ " is assumed which is responsible for the filling of the middlefield-position

(2) a ..., weil er [ $\mathrm{V}_{\text {ATTR }}$ [FOC dir]] versucht $\varnothing$ zu helfen.

and is responsible for topicalisation, too:

$\mathrm{b}\left[\mathrm{V}_{\mathrm{ATTR}}[\right.$ FOC Dir] $]$ versucht er $\varnothing$ zu helfen.

This uniform syntactic treatment of topicalisation and the filling of the focus-position in the middlefield corresponds to the functional similarities of both positions. The focus is the part of the sentence which can contain the new information (Geilfuß 1991) and the filling of the focus-position ("Fokussierung") is a means to make prominent a certain part of the sentence with the background of common information in the discourse of speaker and hearer.

It is proposed in this article that the constructions in question are not-coherent in the sense of Bech (1983). For a sentence like (2)a no form of raising has to be assumed. The infinite embedded sentence is extraposed: no union of matrix-sentence and infinite complement has taken place. 


\section{$1 \quad$ Einleitung}

Der Terminus "verschränkte Konstruktion" (Kvam 1983, auch dritte Konstruktion, siehe Geilfuß (1991) und ebenfalls Hinrichs \& Nakazwa (1998)) bezieht sich auf Sätze mit einem regierenden (finiten) Verb und (mindestens) einem regierten Verb im Infinitiv, in denen ein syntaktisches Komplement des infiniten Verbs vor dem finiten Verb erscheint. Beispiele ((1)f-g aus Geilfuß (1991) sind:

(1) a ..., weil er dieses Auto meinte wiederzuerkennen.

b ..., weil er sie das bittet zu lesen.

c ..., weil er das versucht zu verschweigen.

f ..., wenn er einem Kind versucht das Märchen vorzulesen.

g ..., wenn er das Märchen versucht einem Kind vorzulesen.

$\mathrm{h}$..., wenn er das Märchen einem Kind versucht vorzulesen.

In diesem Aufsatz wird ein transformationeller Erklärungsansatz im Rahmen der Semantischen Syntax (=SeSyn, Seuren 1996) beschrieben, der diese Sätze als inkohärente Konstruktionen im Sinne von Bech (1983) betrachtet. Die NP vor dem Finitum fehlt der VP am Satzende, die ansonsten aber noch intakt ist, wodurch sich Inkohärenz ergibt. Man könnte von "Scrambling" sprechen, einer Links-Bewegung des betreffenden Komplements. Verbprojektions-Anhebung ist für diese Konstruktionen nicht anzunehmen, u.a. deshalb, weil dies ein erklärender Mechanismus unter dem Eindruck von Sprachdaten aus dem Flämischen und auch dem Schweizerdeutschen ist. In diesen beiden Sprachen gilt im Nebensatz die Abfolge Regens vor Rektum. Das Deutsche dagegen weist die spiegelbildliche Anordnung auf.

Für die Besetzung der Fokusposition soll in diesem Aufsatz ein Prädikat angenommen werden, das gleichzeitig für die Vorfeldbesetzung verantwortlich ist. Auch im Vorfeld ist ebenso eine Fokusposition möglich wie im Mittelfeld. ${ }^{1}$ Durch die Annahme eines Prädikats kann syntaktisch dieser Korrespondenz Rechnung getragen werden.

\section{$2 \quad$ Fokusposition im Mittelfeld}

\subsection{Kriterien}

Sehen wir uns nochmals einige Beispiele an:

(2)a ... Diesen Wagen hat Peter versucht zu verkaufen. (Kvam 1983: 114)

b ..., den ich Sie bitte auszufüllen. (ibd.:93)

c ... Ein kleiner Fehler, den wir Sie bitten zu entschuldigen. (GdS 1997: 2196)

d ..., in der man alles meinte kommandieren zu können. (ibd.: 91)

e ..., wenn ich einen solchen Brief beginne zu schreiben. (ibd.)

f Sie haben die neue Grammatik nie versucht an einem Stück zu schreiben. (GdS 1997: 2196)

Die Sätze des Typs (2)a-c zeigen Vorfeldbesetzung: In (2)a steht das direkte Objekt in satzinitialer Stellung, b/c enthalten Relativsätze, wobei das Relativpronomen nach links gewandert ist. (2)d-f sind die Fälle, die uns jetzt erst einmal interessieren sollen. Oben wurde

\footnotetext{
${ }^{1}$ Dieser Gedanke, allerdings nicht näher ausgeführt, findet sich schon bei Abraham (1994: 64).
} 
der Terminus Verschränkung als Oberbegriff für derartige Konstruktionen genannt. Dieser wird in der Grammatik der deutschen Sprache $(=\mathrm{GdS})$ und Kvam präzisiert, wobei neben den Sätzen mit Vorfeldbesetzung und Relativpronomen auch (2)d-f der Untergruppe der Infinitivverschränkungen zugeordnet werden.

In GdS finden wir die folgende Definition (1997: 2195):

Bei den integrierten Konstruktionen mit Infinitivverschränkung sind Teile der IK (=Infinitivkonstruktion, Anm.von mir, M.R.) vor, der Infinitiv selbst (sowie andere Teile) nach - jedoch nicht unbedingt direkt nach - die rechte Satzklammer gestellt. Kennzeichnend für Infinitivverschränkungen ist somit die Nachfeldstellung (Extraposition) des Infinitivs. Aus der Perspektive des extraponierten Infinitivs sind die im Mittelfeld, in der linken Klammer oder im Vorfeld befindlichen Teile der IK linksversetzt.

Ähnlich sieht es Kvam (1983: 6):

[...] eine Größe der eingebetteten Infinitivphrase bzw. des eingebetteten Satzes [steht] vor dem Prädikat, bzw. einem Prädikatsteil des Matrixsatzes. Der Rest der eingebetteten Infinitivphrase bzw. des eingebetteten Satzes folgt kontinuierlich nach dem Matrixsatz. Diese diskontinuierlich links stehende Größe wird Linksverschachtelungsgröße genannt.

Kvam gibt u.a. folgendes Beispiel mit struktureller Aufgliederung (ibd.: 7), wobei "LV" für Linksverschachtelung steht:
(3) ... wenn ich
Ihnen
versuchen darf
ein wenig zu helfen
MATRIXGRÖßEN|LV-GRÖßE
MATRIXPRÄDIKAT
EINGEBETTETE IP
$\div$ LV-GRÖßE

In (3) ist das indirekte Objekt Ihnen die betreffende Linksverschachtelungsgröße. Die Infinitivphrase (=IP) [IP ein wenig zu helfen] ist unvollständig, weil das dazugehörende indirekte Objekt vor dem Matrixprädikat steht. Dass es sich bei Verschränkung um ein syntaktisches Phänomen handelt, das Rückschlüsse auf die idiosynkratischen Eigenschaften der beteiligten Verben erlaubt, zeigt (4):

(4) a ...,weil Hans den Großeltern das Paket versucht/ versucht hat zu bringen. ${ }^{2}$

b ...,weil Hans den Großeltern das Paket hilft/geholfen hat zu bringen.

c(??) ...,weil Hans das Kind dem Großvater bittet zu helfen.

$\mathrm{e}^{*}$...,weil das Kind den Hans der Mutter gebeten hat ein wenig zu helfen.

Bitten hat andere Eigenschaften als z.B. versuchen und helfen, das machen die Beispiele in (4) deutlich. Grewendorf zählt zu der Gruppe von Verschränkung zulassenden Verben neben versuchen auch hoffen, beginnen, anfangen, vergessen, fürchten, (vermögen, beschließen) sowie beabsichtigen (1991: 276) und bemerkt, dass Verben dieser Gruppe kein Akkusativoder Dativ-Objekt subkategorisieren (ibd.).

In Geilfuß (1991: 19ff) findet sich eine Behandlung ähnlicher Strukturen, wobei er Verbprojektionsanhebung als Regel für die deutsche Syntax annimmt. Diese Transformation wurde ursprünglich zur Erklärung von Konstruktionen des Flämischen formuliert:

\footnotetext{
${ }^{2}$ Auch bei der Partizip II-Form eines der betreffenden Verben ist Infinitivverschränkung möglich.
} 


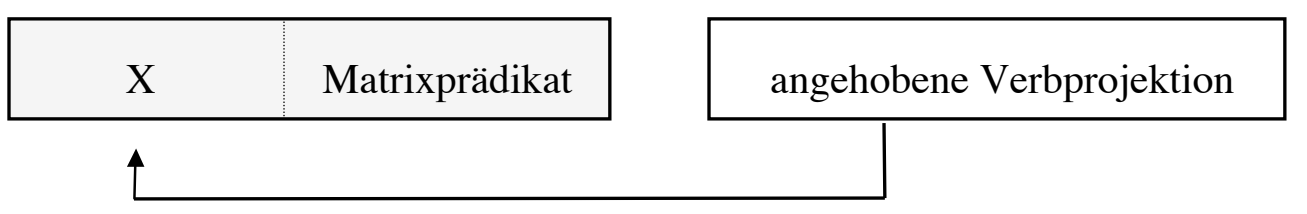

Zurückbewegung (Pseudoscrambling) eines/mehrerer nominalen Elements/nominaler Elemente (X) der Verbprojektion vor das Matrixprädikat.

Abb. 1

Bereits in Richter (2000) wurde bezweifelt, ob die Annahme von Verbprojektionsanhebung für eine Behandlung deutscher Syntax sinnvoll ist, z.B. weil Sätze aus dem Niederländischen wie

(5)a ,dat Marie wilt Jan nen boek kunnen geven. dass Marie will Jan ein Buch können geben. 'dass Marie dem Jan ein Buch geben können will.'

oder aus dem Schweizerdeutschen (von Stechow \& Sternefeld 1988: 413ff):

b das er em Karajan wil chöne en arie vorsinge. dass er dem K. will können eine Arie vorsingen. 'dass er dem K. eine Arie vorsingen können will.'

im Standard-Deutschen einfach nicht gehen. Konstatiert werden kann jedoch, dass Geilfuß, ebenso wie Kvam und GdS die Infinitivverschränkung als eine Zurückbewegung des entsprechenden Elements nach links vor das Matrixprädikat interpretieren, wobei sich Parallelen zu der Analyse von Coppen \& Klein (1992) zur dritten Konstruktion im Niederländischen zeigen. Während Kvam als verschränkbare Elemente auch Adverbiale, Kasusobjekte (direktes/indirektes Objekt), Präpositionalobjekte sowie Prädikative angibt (1983:92f), beschränkt Geilfuß sich auf die Untersuchung der Zurückbewegung von direktem und indirektem Objekt. Kvam geht anders als Geilfuß (s.u.) davon aus, dass sich nur ein Element vor dem finiten Verb befinden kann:

Hauptsätze

(6)a Diesen Mangel versuchten viele durch eine besondere Großzügigkeit auszugleichen.

b Ich möchte auf Ihre präzise Frage versuchen eine präzise Antwort zu geben. (Mittelfeldbesetzung, nach Kvam vorwiegend im mündlichen Sprachgebrauch vorkommend)

Nebensätze

(7)a ... einen kurzen Fragebogen, den ich Sie bitte auszufüllen.

b ..., in der man alles meinte kommandieren zu können. 
Wie auch Geilfuß und GdS interpretiert Kvam Extraposition eines eingebetteten Infinitivs als zweisyntagmatisch, also als inkohärent, wie in er versucht, seinem Bruder zu helfen. Einsyntagmatische Strukturen können Verbalkomplexe aufweisen wie in (8) (Kvam 1983: 192):

(8) Peter möchte seinem Bruder helfen.

In (8) steht das Subjekt auf einer Position, die es in pragmatischem Sinne zum Thema des Satzes macht. Diese Position - in Kvam findet sich auch der Terminus Fundamentstelle - kann auch durch andere Satzglieder besetzt werden, die eigentlich aus einer eingebetteten Infinitivphrase stammen. Vor dem Einsetzen der betreffenden Transformation sieht die Struktur folgendermaßen aus (Kvam 1983: 192):

Fundament

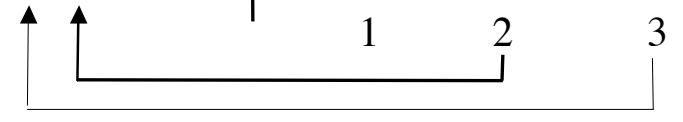

\author{
Schlußfeld \\ helfen \\ 4
}

Abb.2

Wenn das indirekte Objekt die Fundamentstelle besetzt, lautet der Satz

(8) Seinem Bruder möchte Peter helfen.

Grewendorf interpretiert (1991) Verschränkungen als kohärente (restrukturierte) Konstruktion mit Verbalkomplex. Das Besondere ist das nicht-satzfinale Finitum im Nebensatz. So wird ein Satz wie

(9) ..., weil der Lehrer das Theorem versucht zu beweisen.

über einen Kamm geschoren mit kohärenten (Neben-) Sätzen mit Verbalkomplex, in denen das Finitum (werden, haben) nicht am Satzende steht, was aus der Literatur als Oberfeldumstellung bekannt ist (siehe Bech 1983).

Oberfeldumstellung berschränkt sich nach den Besten \& Broekhuis (1992:25) unter Verweis auf Geilfuß nicht auf haben und werden (die Grammatikalitätsbewertungen stammen von mir, M.R.):

(10)a $\quad$..., weil das Wetter scheint schön zu werden.

b(??) $\quad$..., dass er noch muss nach Bonn zurückfahren können.

$c(? ? / *) \quad$..., dass er möchte wieder Museen besuchen können.

$\mathrm{d}\left(? ?\right.$ ?* $^{*} \quad$..., dass er es doch muss machen können.

(10)c zum Beispiel enthält in der Tat eine angehobene Verbprojektion - der Satz jedoch ist in allerbestem Fall äußerst zweifelhaft, wahrscheinlich aber ist er schlichtweg ungrammatisch.

In GdS (1997:2196) wird die Restrukturierungshypothese Grewendorfs für die verschränkten Konstruktionen mit dem Argument abgewiesen, dass rechts vom Finitum die Komplexität des möglichen Materials wesentlich begrenzter sei als bei Verbindungen von Verben wie 
versuchen, bitten ...., bzw. liege in der Transparenz der Unterschied zwischen den VerbClustern:

(11)a Sie haben die neue Grammatik nie versucht an einem Stück zu schreiben. vs.

b* ..., dass er hat immer für seine Mutter arbeiten müssen.

GdS zufolge (ibd.) ist bei Verschränkungen kein Verbalkomplex möglich und somit ist die Konstruktion nicht kohärent. Dies bedeutet, dass man nicht von einer einheitlichen Erklärung für Sätze des Typs (11)a und (11)b ausgehen kann, auch wenn man (11)b ersetzt durch das grammatische... dass er immer für seine Mutter hat arbeiten müssen. Dieser Satz ist kohärent, die Struktur des Verbalkomplexes kann in etwa so wiedergegeben werden:

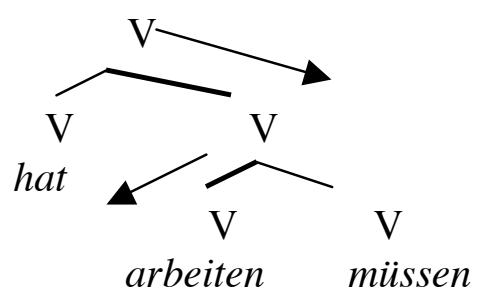

Abb.3

Die Verzweigungsrichtung ist, wie man sieht, hybrid: Im unteren Teil des Komplexes verzweigt der Komplex sich nach links, im oberen nach rechts. Noch deutlicher wird dies anhand des Strukturbaum für wird haben arbeiten dürfen sollen:

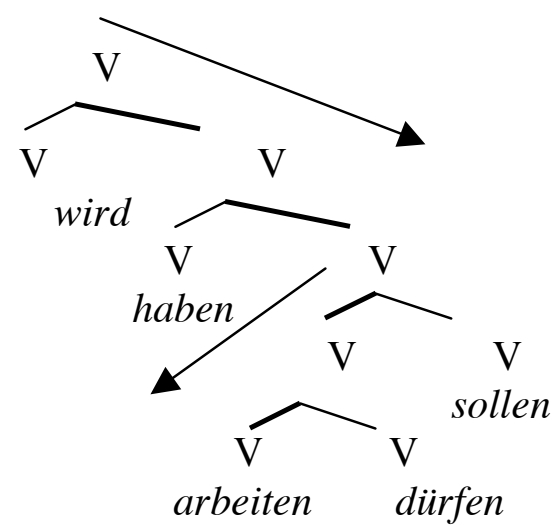

Abb. 4

Der Analyse für Sätze des Typs (11)a soll nicht vorweggegriffen werden, so viel kann aber gesagt werden, dass es sich um zwei verbale Gruppen (hat versucht und zu schreiben) in zwei Satzstrukturen mit Subjektkontrolle handelt. Diese Sätze enthalten keinen Verbalkomplex und sind somit inkohärent.

\subsection{Kennzeichen des Fokus}

Auch Vorfeldbesetzung interpretiert Kvam als eine Variante der verschränkten Konstruktion. Wo auch immer das betreffende Element landet, es ist das Objekt einer 
Thematisierungstransformation (1983: 190). D.h. das versetzte Element wird im Sinne einer kommunikativ-pragmatischen Strukturierung Thema des Satzes und befindet sich in Ausdrucksstellung. (Schmidt 1967 zit. nach Kvam 1983: 195) Der entsprechende Satzteil gilt im Diskurs als bekannt oder ableitbar (ebd.: 186), fügt diesem keine neue Information hinzu und darf vor dem Finitum stehen, unter Umständen in satzinitialer Position.

Anders sieht es Geilfuß, der zurückversetzte Elemente als Teile einer Fokusprojektion interpretiert. (1991: 22ff). Fokus ist der die neue Information enthaltende Satzteil, der deswegen eine besondere Stellung im Satz erhalten kann (Lewandowski 1990: 306). Dies kann die Stellung links vom Finitum sein. Fokussierung ist nach GdS (1997: 232) ein pragmatisch-kontextuelles Verfahren zur Hervorhebung von bestimmten Teilen einer Aussage. Das fokussierte Element, das u.a. die für den Hörer neue Information ausdrücken kann (GdS 1997: 233), erhält sein kontextuelles Gewicht durch und den Bezug auf bereits vorhandene Information, bzw. Wissen. Mittels Echofragen kann nach Geilfuß getestet werden, welches Satzteil Fokus ist, bzw. sein kann, jedoch müssen die Antworten "passend" sein. (1991: 22ff).

In Großbuchstaben geschriebene Wörter sind betont. Unter iv findet sich die Echofrage nach dem Fokus im Vorfeld und auch die "passende" Antwort:

\section{Frage}

i ... ein Fehler, den ich WEN bitte zu entschuldigen?

ii ... weil der Lehrer WAS versucht zu beweisen?

iii ... wenn ich WEM versuchen darf ein wenig zu helfen?

iv ... WAS backst du?

\section{Antwort}

i ... ein Fehler, den ich SIE bitte zu entschuldigen.

ii ... weil der Lehrer DAS THEOREM versucht zu beweisen.

iii ... wenn ich IHNEN versuchen darf ein wenig zu helfen.

iv ... EINEN KUCHEN backe ich.

Die Antworten sind jeweils wohlgeformt, damit ist nach Geilfuß erwiesen, dass die vor das Finitum gerückten, zum eingebetteten Infinitiv gehörenden Elemente fokusfähig sind. Auffällig ist, dass die Fragesätze dem natürlichen Sprachgebrauch eher entsprechen als die Antwortsätze, wobei die Interrogativpronomen jeweils auch auf der kanonischen Position vorkommen können (..., weil der Lehrer versucht, WAS zu beweisen?).

Der zurückbewegte Satzteil verharrt rechts vom Subjekt, es sei denn, dieses ist kein pronominales Subjekt und jenes ein klitisches Objekt: ..., weil es die Kinder versuchen zu essen.

Bei der Zurückbewegung von zwei Satzteilen Geilfuß (1991) wie in

$$
\begin{aligned}
& \text { (13)a } \ldots, \text { weil ich }\left[\mathrm{NP}_{\text {indirektes Obj }} \operatorname{dir}\right]\left[\mathrm{NP}_{\text {direktes Objekt }} \text { die Problematik }\right] \text { versuche } \\
& \text { darzulegen } \\
& \text { b(?)..., weil ich }\left[\mathrm{NP}_{\text {direktes Objekt }} \text { die Problematik }\right]\left[\mathrm{NP}_{\text {indirektes Objekt }} \text { dir }\right] \text { versuche }
\end{aligned}
$$


darzulegen

zeigt (13)a die unmarkierte Abfolge der zurückbewegten Satzteile, hier befindet sich das indirekte vor dem direkten Objekt. Die Abfolge in (13)b ist markiert. Beide NPs können unabhängig voneinander intonatorisch hervorgehoben werden, bzw. trägt ein Satzteil sehr nachdrücklich den Hauptakzent, während in (13)a die Objekte in unmarkierter Abfolge eine Fokussierung erfahren, nach Geilfuß "pseudo"-gescrambelt sind. ${ }^{3}$ Wie skopustragende Elemente in diese Konstruktion integrierbar sind, kann anhand der Negation deutlich gemacht werden:

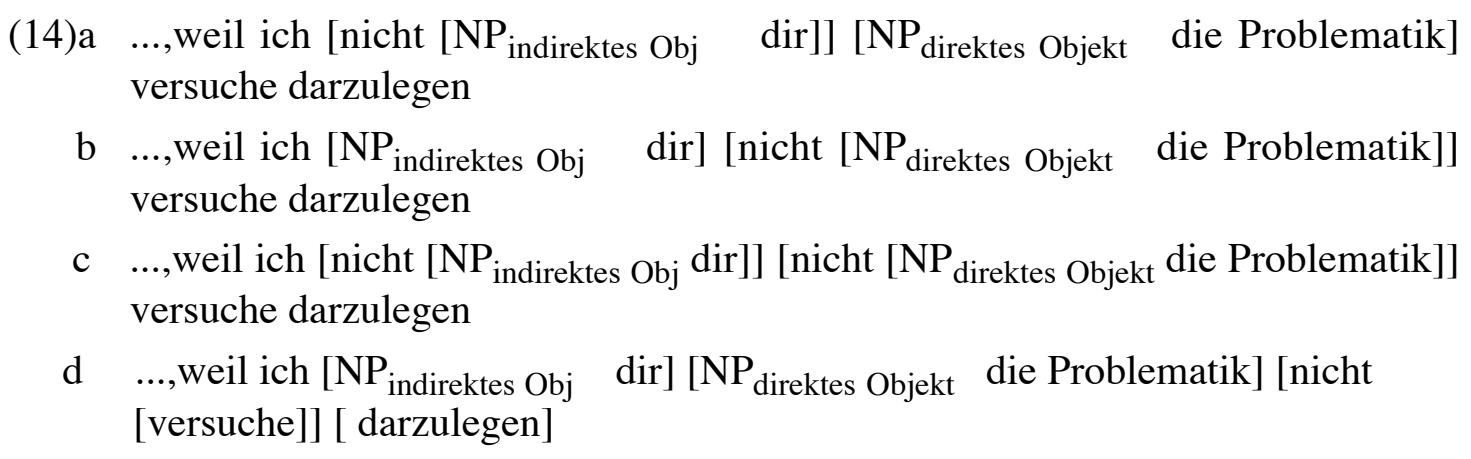

Nur in (14)d befindet sich der gesamte Satz im Skopus der Negation, die damit in der Tiefenstruktur die hierarchisch höchste Position einnimmt. In den anderen Beispielsätzen sind lediglich jeweils die zurückbewegten Satzteile im Bereich der Negation.

\subsection{Eine einheitliche syntaktische Behandlung}

Vorfeldbesetzung ist nicht das eigentliche Thema dieses Aufsatzes. Dennoch sollen ein paar Worte darüber verloren werden, wie die Verschiebung von, in diesem Fall, nominalem Material ins Vorfeld geregelt ist innerhalb der transformationellen SeSyn, einer generierenden Grammatik,. Es wird sich dann zeigen, dass Mittelfeldfokus-Besetzung auf dieselbe Art und Weise funktioniert. Somit entspricht die syntaktische Behandlung der FunktionsÜbereinstimmung der fraglichen Positionen in Vor- und Mittelfeld: Sie können als Position für den Satzfokus dienen.

Die Topikalisierungsregel - Seuren nennt sie Fronting (1996: 284ff) - wird ausgelöst durch das Prädikat $\mathrm{V}_{\text {ATTR }}$ mit der Einsetzungsoption [Foc] (ibd.: 281). Die mögliche, vereinfachte ${ }^{4}$, Struktur eines Satzes mit einer Fokus-Position im Vorfeld und vor der Fokussierungstransformation ist die folgende:

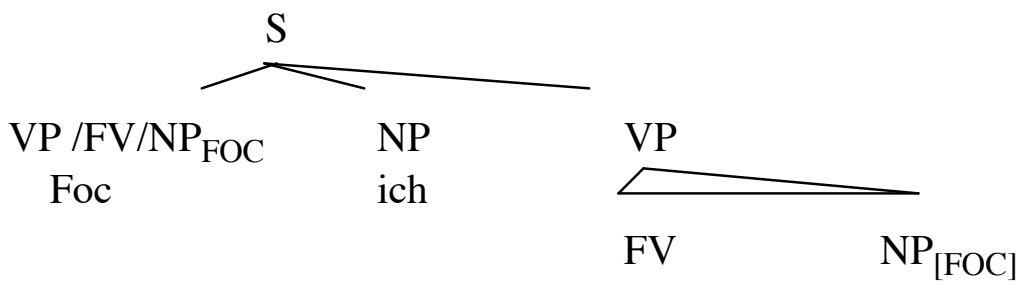

\footnotetext{
${ }^{3}$ Geilfuß gibt als Kriterium für Scrambling an, dass die zurückbewegten Elemente nicht die "default"Reihenfolge beibehalten. Läßt die Zurückbewegungstransformation die ursprüngliche Abfolge intakt, liegt nach Geilfuß Pseudocrambling vor (1991: 20ff).

${ }^{4}$ Weggelassen sind z.B. Tempusprädikate.
} 
backe

einen Kuchen

Abb.5

$\mathrm{V}_{\text {ATTR }}$ bezeichnet eine Menge von Prädikaten: Je nach zu erzeugender Struktur verändert sich $\mathrm{V}_{\mathrm{ATTR}}$ in eine bestimmte Kategorie. Im Beispiel EINEN KUCHEN backe ich ist dies die kategorialgrammatisch anmutende Kategorie $\mathrm{VP} / \mathrm{FV} / \mathrm{X}_{\mathrm{FOC}}$. Zuerst muß irgendein Element aus der Menge der Töchter von S mit dem Feature "Foc" sich der Links-Bewegung unterziehen, ausgelöst durch VP/ FV/X $\mathrm{X}_{\mathrm{FOC}}{ }^{5}$, dann FV, das finite Verb. Hiermit wird der Verb-Zweitstellung im Deutschen Rechung getragen. Der ganze topikalisierte Komplex in unserem Beispiel einen Kuchen backe ich ist die VP [ ${ }_{\mathrm{VP}}$ einen Kuchen backe]:

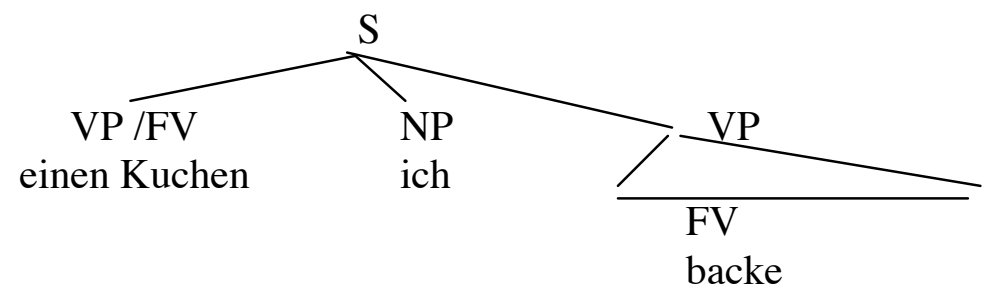

a

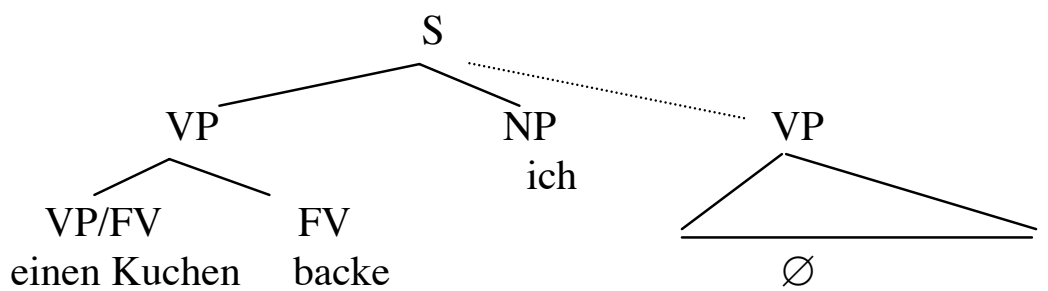

b

Abb.6

Übertragen wir diese Regeln auf die verschränkten Konstruktionen. Zuerst einmal soll eine Struktur ohne Fokussierung generiert werden, etwa weil ich versuche, die Problematik darzulegen oder weil ich die Problematik darzulegen versuche, um ein wenig Vertrautheit mit der SeSyn-Grammatik zu erlangen. Die Tiefenstruktur - es gilt eine V-S-O-(Prädikat-SubjektObjekt) Abfolge - ist folgendermaßen ${ }^{6}$

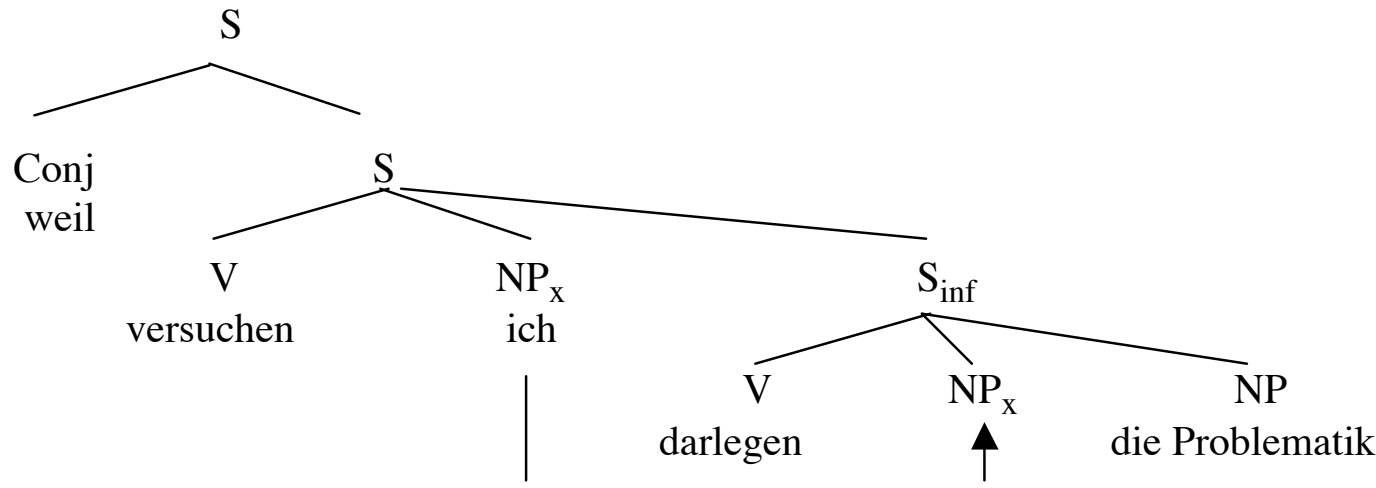

\footnotetext{
${ }^{5}$ In Seuren (1996) ist die Reihenfolge der anzubindenden Elemente und auch die Neigungsrichtung der SlashSymbole genau anders herum: $\mathrm{X}_{\mathrm{FOC}} / \mathrm{FV} / \mathrm{VP}$.

${ }^{6}$ Seuren (1996:24f.) beruft sich dabei auf McCawley (1970), der V-S-O / S-O-V für alle Sprachen annimmt, da diese Hypothese die höchst mögliche Ökonomie in Ableitung oder Generierung gewährleistet. Seuren interpretiert das Deutsche als V-S-O Sprache, die in einem früheren Stadium eine S-O-V Abfolge hatte. Er räumt ein (persönliche Mitteilung), dass S-O-V auch für das heutige Deutsche eine erwägenswerte Alternative sei.
} 


\section{Abb.7}

Subjekt-Kontrolle

Nach Tilgung des leeren, kontrollierten Subjekts in $\mathrm{S}_{\text {inf }}$, Links-Bewegung (Anhebung) des Subjekts im Matrixsatz und $z u$-Einsetzung in der $\mathrm{VP}^{2}$ (ausgelöst durch Tilgung des Subjekts) ergibt sich folgende Struktur, wobei die Regeln bottom-up wirken ${ }^{7}$ :

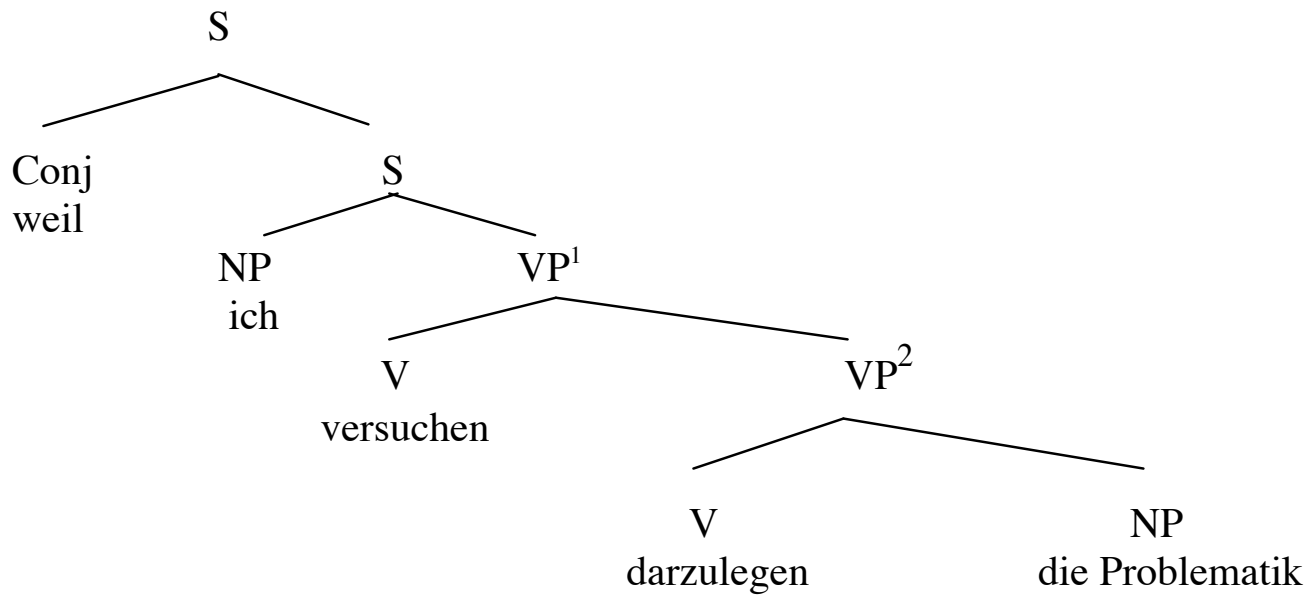

Abb.8

Auf diese Struktur wirkt jetzt die Regel V-Final ein, die in Hauptsätzen den nicht-finiten Teil des V-Clusters ans Satzende versetzt und in Nebensätzen den gesamten V-Cluster. Es gibt jetzt zwei Möglichkeiten für die V-Final-Regel, die jeweils den beiden Sätzen weil ich versuche, die Problematik darzulegen und weil ich die Problematik darzulegen versuche entsprechen.

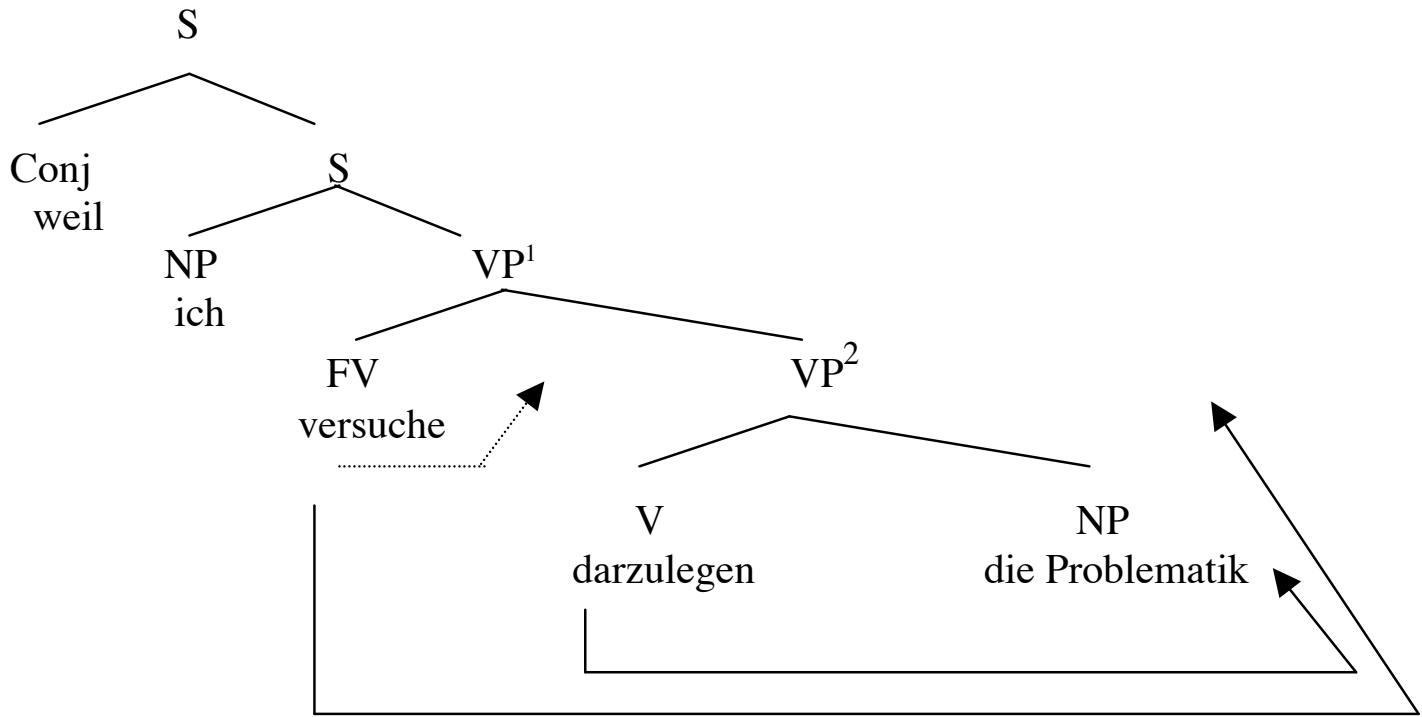

Abb.9

7 Diese Regeln werden teils durch lexikale Features ausgelöst (Subjekt-Tilgung), teils aber auch strukturell (Subjektanhebung). Genaueres erfährt man in Seuren (1996). Um den Leser nicht zu verwirren, sind in diesem Aufsatz die lexikalischen Features fast ausnahmslos weggelassen worden. 
Die Pfeile zeigen die Optionen der Bewegung V-Final an, die den gesamten V-Komplex (im Nebensatz), bzw. den nicht-finiten Teil des V-Komplexes (im Hauptsatz) nach rechts rückt. Die Struktur verdeutlicht auch, dass versuchen mit dem regierten Prädikat keinen Verbalkomplex bildet. Das Matrixprädikat versuchen wird also entweder phrasenfinal und bleibt links von der eingebetteten VP (gestrichelte Linie), oder aber es wird satzfinal und landet rechts von der eingebetteten VP (dünne, durchgezogene Linie). Im ersten Fall erhalten wir weil ich versuche, die Problematik darzulegen im zweiten Fall weil ich die Problematik darzulegen versuche.Verbprojektions-Anhebung ist in dieser Analyse nicht notwendig.

Jetzt aber zu der Zurückbewegung von nominalem Material auf die Fokusposition im Mittelfeld. Die fokussierten Elemente, so soll die Anfangsüberlegung sein, landen in der VP mit dem Matrixprädikat. Wie die bisherigen Ausführungen jedoch zeigten, handelt es sich nicht um eine kohärente, monosententiale Struktur, sondern um einen inkohärenten Satz. Die zurückbewegten Elemente landen auf einer Fokusposition in der höheren VP.

Wir wählen als Beispiel einen Satz mit zwei zurückbewegten nominalen Elementen wie in (15)

(15)a ..., weil er dir die Problematik versucht darzulegen.

und sehen uns an, wie im SeSyn-Stil eine Tiefenstruktur aussehen könnte.

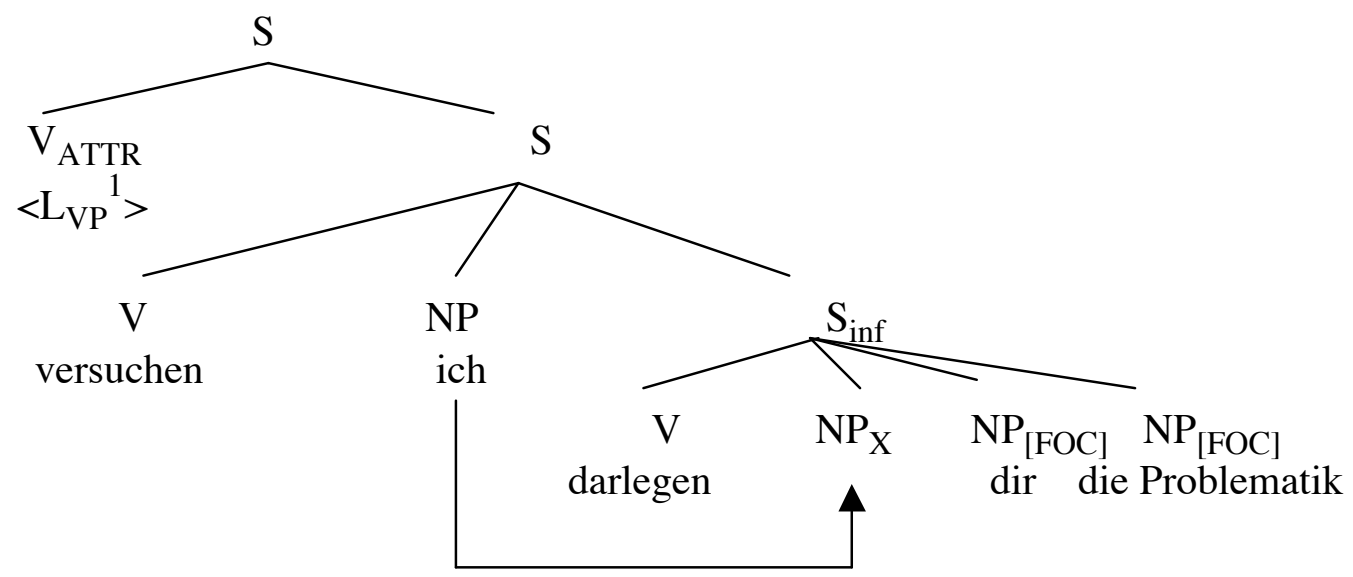

Subjekt-Kontrolle

Abb. 10

Wie oben gesagt (siehe Abb. 6) hat in der Tiefenstruktur jeder von S regierte Teilbaum die Anordnung Prädikat-Subjekt-\{Objekt $\}$ (V-S-O). Das Prädikat $\mathrm{V}_{\text {ATTR }}$ hat jetzt das Rulefeature $\mathrm{L}_{\mathrm{VP}}{ }^{1}$, was bedeutet, dass eine Rechtsbewegung ( $\mathrm{L}=$ Lowering) von $\mathrm{V}_{\mathrm{ATTR}}$ stattfinden muss, und zwar in die $\mathrm{VP}^{1}$, direkt rechts neben das Matrixprädikat. Lassen wir wieder die Regeln in bottom-up-Richtung ihre Arbeit beginnen. Die nächste Ableitungsstation erinnert an die Struktur in Abbildung 7: 


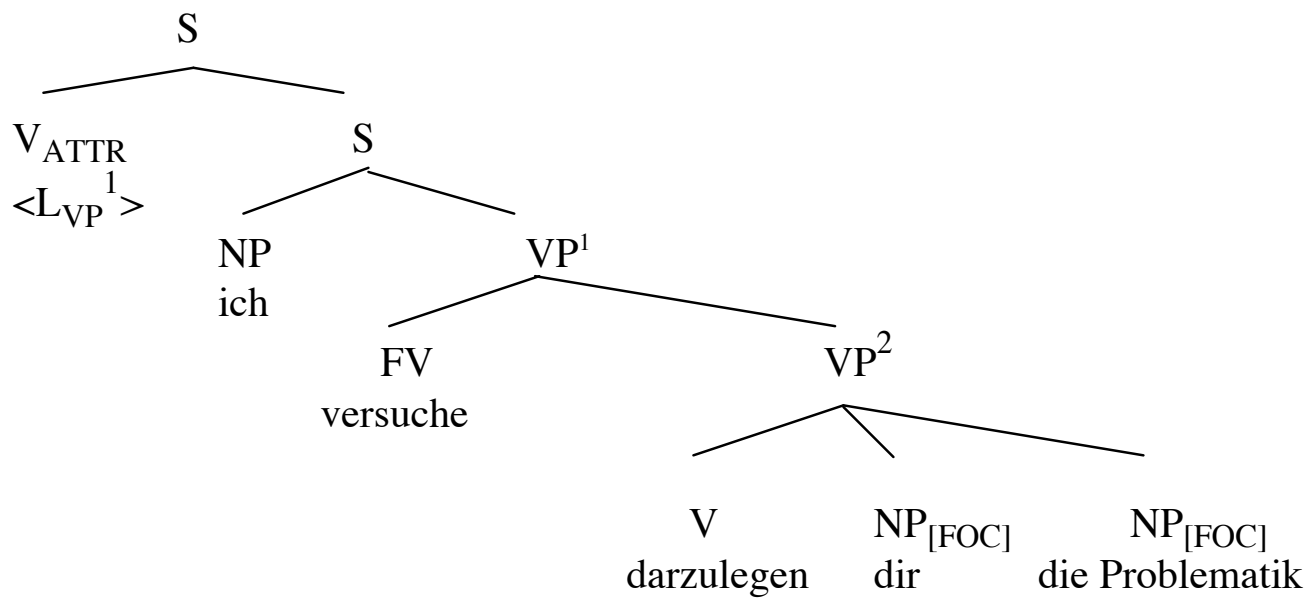

Abb.11

Als nächstes muss das Fronting-Prädikat nach rechts bewegt werden und zwar in die $\mathrm{VP}^{1}$ und verwandelt sich in $\mathrm{FOC} / \mathrm{NP}_{[\mathrm{FOC}]} / \mathrm{NP}_{[\mathrm{FOC}]}$ (siehe Fußnote 6).:

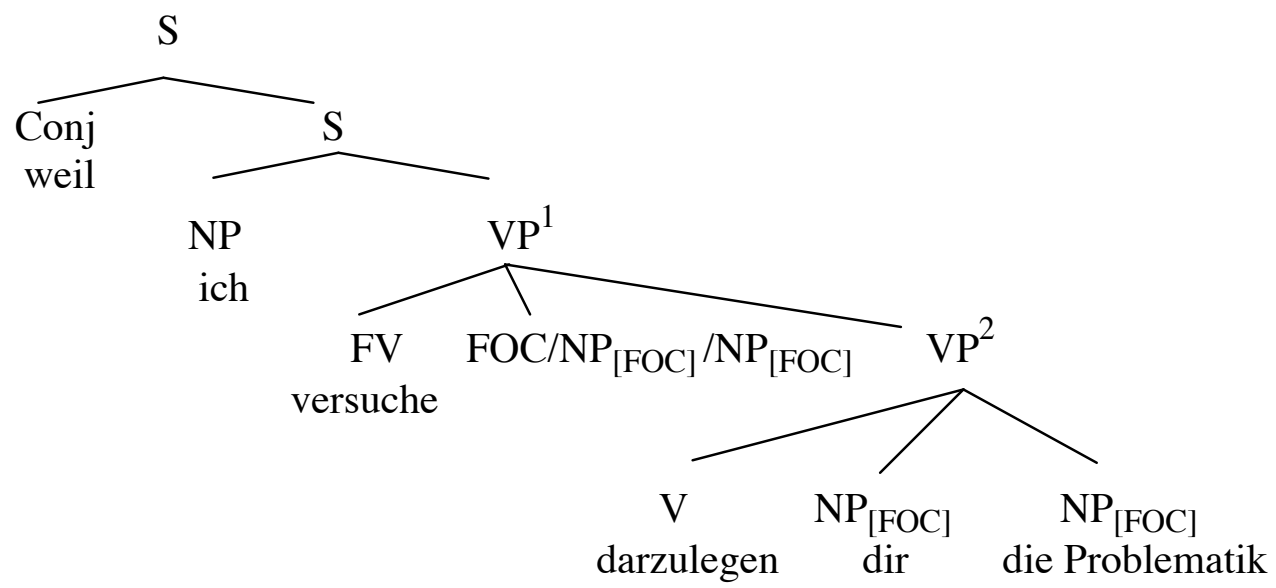

Abb. 12

Mit einer Applikation im Sinn einer Regel der Kategorialgrammatik rückt der erste FokusKandidat nach links:

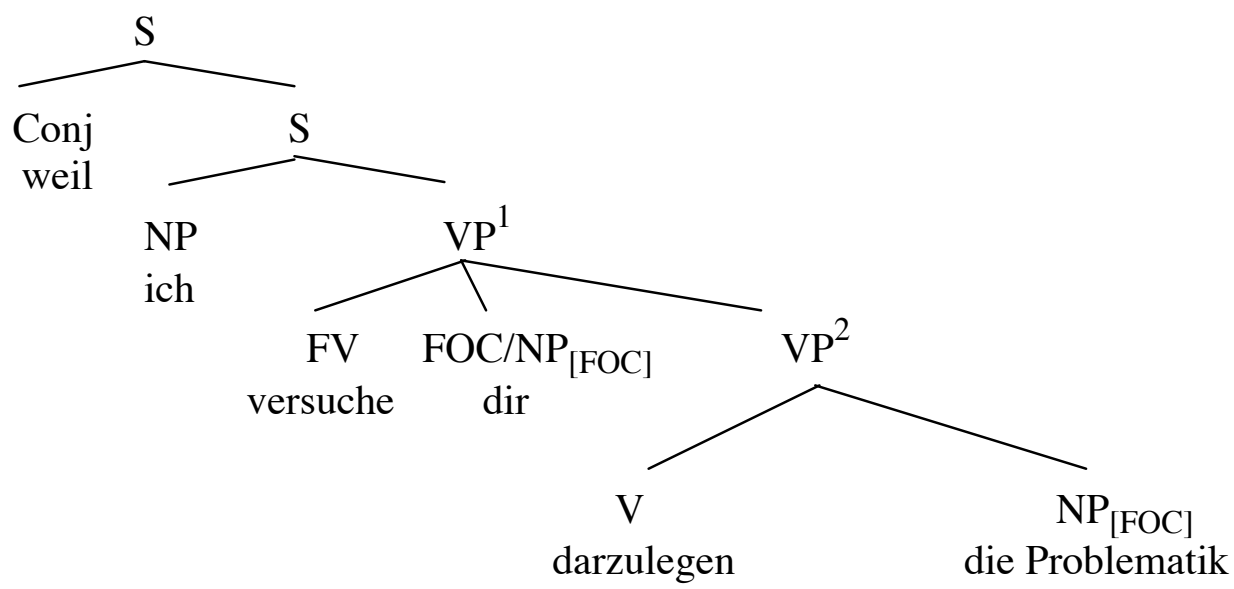

Abb.13 
Das zweite Fokuselement rückt nach:

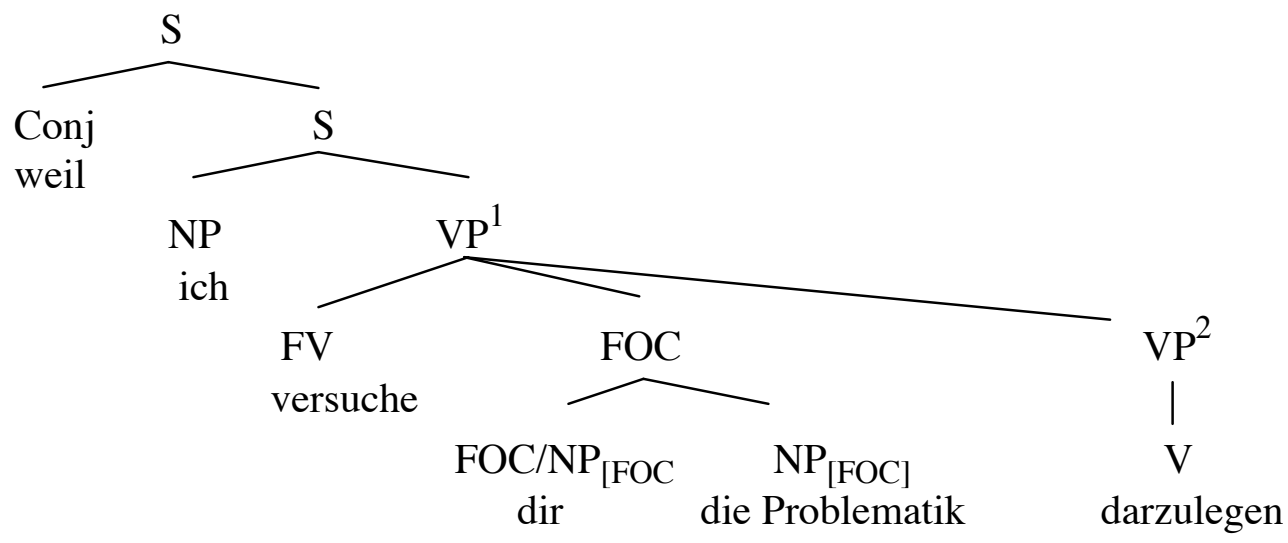

Abb.14

Jetzt sorgt die Regel V-Final, die, wie wir uns erinnern, im Hauptsatz den nicht-finiten Teil des V-Clusters und im Nebensatz den gesamten V-Cluster ans Satzende transportiert, für die gewünschte (Beinahe-)Oberflächenstruktur:

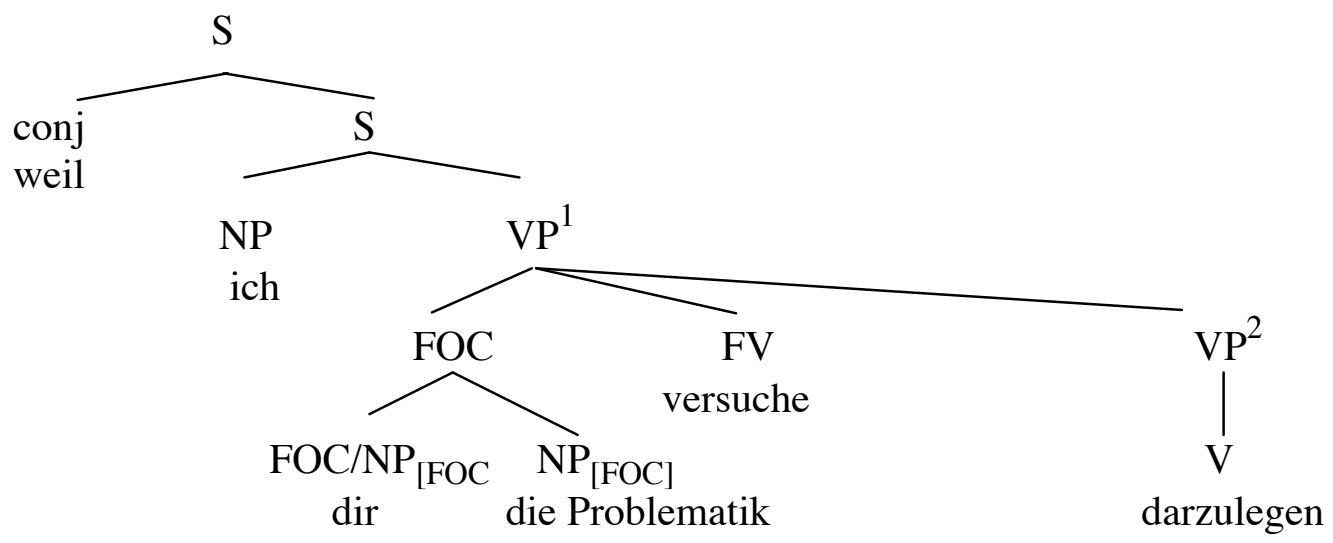

Abb. 15

Die eben vorgeschlagene Analyse für Verschränkungen in Nebensätzen ist auch brauchbar in Hauptsätzen, wie in

(16)a Dir versuche ich die Problematik darzulegen.

b Die Problematik versuche ich dir darzulegen.

c Dir die Problematik versuche ich darzulegen.

d Ich werde dir die Problematik versuchen dazulegen.

Die Sätze (16)a-c weisen NPs im Vorfeld auf. Sie haben das Merkmal "[FOC]" und wurden durch das Prädikat $\mathrm{V}_{\text {ATTR }}$ nach links gezogen, man vergleiche hierzu noch einmal Abb. 5 und

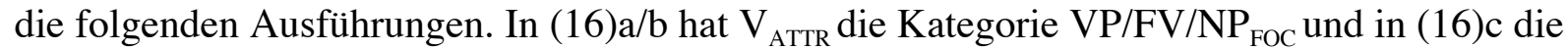
Kategorie $\mathrm{VP} / \mathrm{FV} / \mathrm{NP}_{[\mathrm{FOC}]} / \mathrm{NP}_{[\mathrm{FOC}]}$. In jedem Fall aber rückt das Finitum mit. Dies ist in Nebensätzen und in Hauptsätzen des Typs (16)d anders. Man könnte die Regel formulieren, dass in keinem Fall, wenn das für Fokussierung zuständige Prädikat $\mathrm{V}_{\mathrm{ATTR}}$ das Feature $\left\langle\mathrm{L}_{\mathrm{VP}}\right\rangle$ 
hat, also nach rechts in eine VP bewegt wird, die resultierende Kategorie _ / FV/ _ sein darf. Der liegende Strich bezeichnet eine Variable, _ / FV/ _ ist eine Kategorie, die auf jeden Fall irgendwann zur Saturierung das Finitum benötigt. Das ist aber in Nebensätzen mit Infinitivverschränkungen und Sätzen des Typs (16)d nicht notwendig, bzw. gar nicht möglich, da sich das Finitum (FV) links von dem Fokus-Prädikat (-Kategorie) befindet.

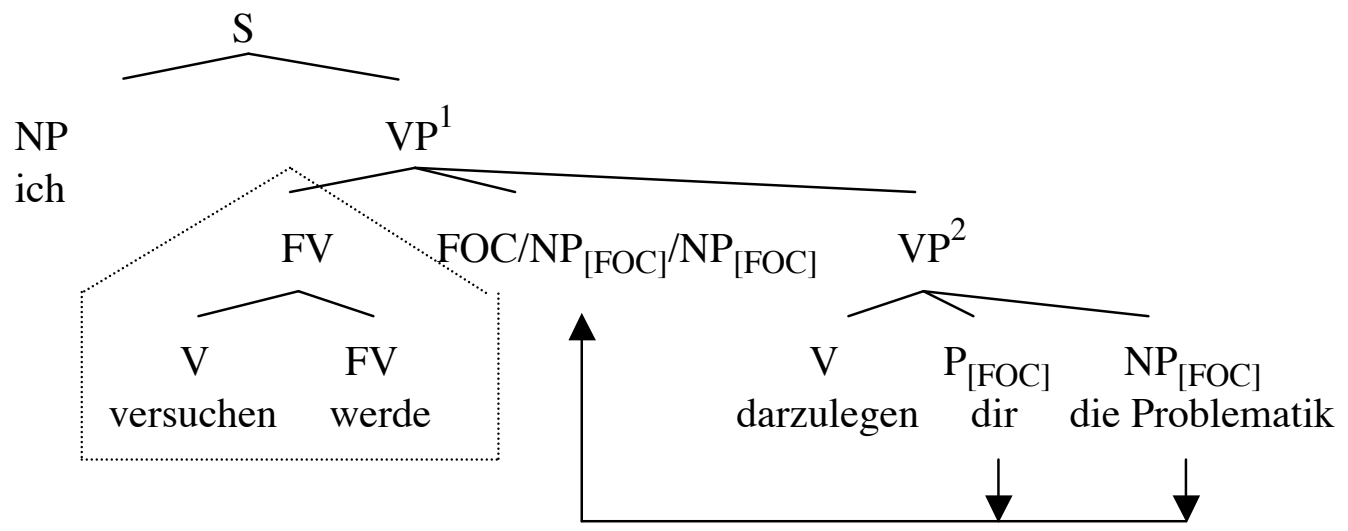

Abb.16

Wenn die Struktur in Abb. 16 zu einem Hauptsatz führen soll, so ergeben sich durch V-Final mehrere Möglichkeiten:

(17)a Ich werde dir die Problematik versuchen darzustellen.

(versuchen als infiniter Teil des V-Clusters wurde an das Ende von VP ${ }^{1}$ versetzt; VFinal in $\mathrm{VP}^{2}$ ist nicht sichtbar, da die beiden nominalen Elemente ja extrahiert wurden)

b Ich werde dir die Problematik darzustellen versuchen.

(versuchen wurde an das Ende des gesamten Satzes versetzt; dieser Satz wäre aber auch ohne Mittelfeldfokus möglich: Wenn aus $\mathrm{VP}^{2}$ kein nominales Material extrahiert würde, so ergäbe $\mathrm{V}$-Final in $\mathrm{VP}^{2}$ :

$\left[\begin{array}{lllll}\mathrm{S} & \varnothing & {[\mathrm{VP}} & \varnothing & {[\mathrm{NP}} \\ \mathrm{dir}\end{array}\right.$ [NP $_{\mathrm{N}}$ die Problematik] [V darzulegen]] [versuchen]

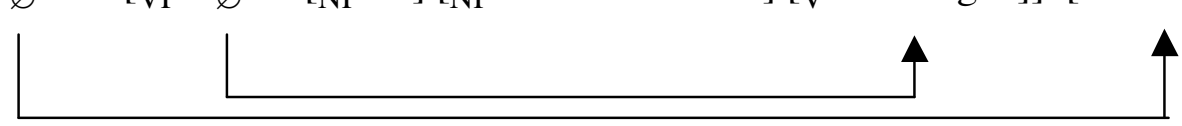

Abb.17

\section{$3 \quad$ Zusammenfassung}

Die hier vorgestellte Semantische-Syntax-Analyse vermeidet die Annahme einer angehobenen Verbprojektion. Es zeigte sich, dass Sätze mit Verschränkungen zweisyntagmatisch, also inkohärent, sind; die eingebettete VP bleibt erhalten, und es entsteht kein Verbalkomplex. Die aus dem eingebetteten infiniten Komplement extrahierten Elemente wurden als mögliche Satzfokusse interpretiert. Für den Mittelfeld-Fokus wie auch die Vorfeldbesetzung, ebenfalls eine mögliche Fokussierungsoperation, wurde nur ein Prädikat angenommen. Diese uniforme syntaktische Behandlung entspricht der pragmatischfunktionalen Korrespondenz der Fokusse in Vor-, bzw. Mittelfeld. 


\section{Literaturangaben}

Abraham, Werner (1994): "Infinitivergänzungen". In: Streube, Anita / Zybatow, Gerhild (Hgg.): Zur Satzwertigkeit von Infinitiven und Small Clauses. Tübingen: 51-74. (=Linguistische Arbeiten 315).

Bech, Gunnar (1983): Studien über das deutsche Verbum infinitum. 2. Auflage. Tübingen.

Besten, Hans den / Jerold A.Edmondson (1983): "The verbal complex in Continental West Germanic". In: Abraham, Werner (Hrsg.): On The formal syntax of the Westgermania. Amsterdam. (=Linguistik Aktuell. Amsterdamer Beiträge zur theoretischen \& angewandten Linguistik. Bd. 3).

den Besten, Hans / Broekhuis, Hans (1992): "Verb Projection Raising in het Nederlands". Spektator 1992: 21-34.

Coppen, Peter-Arno / Klein, Maarten (1992): "The End of V-raising". In: Nieuwe Eskapades in de Neerlandistiek. Een bundel opstellen voor M.C. van den Toorn. Groningen: 32-46.

Geilfuß, Jochen (1991): "Verb- und Verbphrasensyntax". In: Sprachtheoretische Grundlagen für die Computerlinguistik. Arbeitspapiere des Sonderforschungsbereichs 340, Bericht Nr. 11. Stuttgart: Wissenschaftliches Zentrum der IBM Deutschland.

Grewendorf, Günther (1991): Aspekte der deutschen Syntax. Eine Rektions-Bindings-Analyse. 2. Auflage. Tübingen.

Hinrichs Erhard W. / Nakazawa, Tsuneko (1998): "Third Construktion and VP Extrapostion in German: An HPSG Analysis". In: Hinrichs, Erhard / Kathol, Andreas / Nakazawa, Tsuneko (Hrsg.): Syntax And Semantics. Complex Predicates in Nonderivational Syntax. Vol. 30. SanDiego: 115- 157.

Institut für deutsche Sprache (1997): Grammatik der deutschen Sprache (in 3 Bänden). Berlin / New York.

Kvam, Sigmund (1983): Linksverschachtelung im Deutschen und Norwegischen. Eine kontrastive Untersuchung zur Satzverschränkung und Infinitivverschränkung in der deutschen und norwegischen Gegenwartssprache. Tübingen (=Linguistische Arbeiten, Nr. 130).

Lewandowski, Theodor (1990): Linguistisches Wörterbuch (in 3 Bänden). Heidelberg / Wiesbaden.

McCawley, James D.(1970): "English as a VSO-Language". Language 46.2: 286-299.

Richter, Michael (2000): Verbkonstruktionen im Deutschen. Eine transformationelle Analyse syntaktischer Erscheinungen innerhalb des deutschen Verbsystems im Rahmen der semantischen Syntax. Dissertation. Universität Nimwegen.

Schmidt, Wilhelm (1967): Grundfragen der deutschen Grammatik. Eine Einführung in die funktionale Satzlehre. 3. Auflage. Berlin.

Seuren, Pieter (1996): Semantic Syntax. Oxford.

Stechow von, Arnim / Sternefeld, Wolfgang (1988): Bausteine syntaktischen Wissens. Ein Lehrbuch der generativen Grammatik. Opladen. 